\title{
Definitions, components and processes of data harmonisation in healthcare: a scoping review
}

\author{
Bey-Marrié Schmidt ${ }^{1,2^{*}}$, Christopher J. Colvin ${ }^{2,3,4}$, Ameer Hohlfeld ${ }^{1}$ and Natalie Leon ${ }^{3,5}$
}

\begin{abstract}
Background: Data harmonisation (DH) has emerged amongst health managers, information technology specialists and researchers as an important intervention for routine health information systems (RHISs). It is important to understand what $\mathrm{DH}$ is, how it is defined and conceptualised, and how it can lead to better health management decision-making. This scoping review identifies a range of definitions for $\mathrm{DH}$, its characteristics (in terms of key components and processes), and common explanations of the relationship between $\mathrm{DH}$ and health management decision-making.

Methods: This scoping review identified relevant studies from 2000 onwards (date filter), written in English and published in PubMed, Web of Science and CINAHL. Two reviewers independently screened records for potential inclusion for the abstract and full-text screening stages. One reviewer did the data extraction, analysis and synthesis, with built-in reliability checks from the rest of the team. We developed a narrative synthesis of definitions and explanations of the relationship between $\mathrm{DH}$ and health management decision-making.

Results: We sampled 61 of 181 included to synthesis definitions and concepts of DH in detail. We identified six common terms for data harmonisation: record linkage, data linkage, data warehousing, data sharing, data interoperability and health information exchange. We also identified nine key components of data harmonisation: DH involves (a) a process of multiple steps; (b) integrating, harmonising and bringing together different databases (c) two or more databases; (d) electronic data; (e) pooling data using unique patient identifiers; and ( $f$ ) different types of data; (g) data found within and across different departments and institutions at facility, district, regional and national levels; (h) different types of technical activities; (i) has a specific scope. The relationship between DH and health management decision-making is not well-described in the literature. Several studies mentioned health providers' concerns about data completeness, data quality, terminology and coding of data elements as barriers to data utilisation for clinical decision-making.
\end{abstract}

Conclusion: To our knowledge, this scoping review was the first to synthesise definitions and concepts of DH and address the causal relationship between $\mathrm{DH}$ and health management decision-making. Future research is required to assess the effectiveness of data harmonisation on health management decision-making.

Keywords: Data harmonisation, Health information exchange, Health information system, Scoping review

\footnotetext{
* Correspondence: bey-marrie.schmidt@mrc.ac.za

${ }^{1}$ Cochrane South Africa, South African Medical Research Council, Francie Van

Zijl Dr, Parow Valley, Cape Town 7501, South Africa

${ }^{2}$ Division of Social and Behavioural Sciences, School of Public Health and

Family Medicine, University of Cape Town, Cape Town, South Africa

Full list of author information is available at the end of the article
} 


\section{Background}

Data harmonisation (DH) in healthcare is a digital, technology-based innovation that can potentially help routine health information systems (RHISs) function at their best. It can help organise and integrate large databases containing routine health information [1]. Designing, developing and implementing $\mathrm{DH}$ interventions has the potential to strengthen aspects of the health system, by enhancing RHISs to high-quality and relevant information that can support decisions, actions and changes across all components and levels of the health system [2, 3]. When RHISs are functioning properly, they can help health practitioners and managers identify and close gaps in health service delivery as well as inform their planning, implementation and monitoring of interventions $[4,5]$. They can also help deal address problems related to using different variables and indicators for collecting, analysing and reporting health information across programmes [6], which is common in low-andmiddle-income (LMIC) settings. Other challenges to effective RHIS functioning include the production of poor-quality data that cannot easily be exchanged and programmatic fragmentation across levels of the health system, which can result in the duplication and excessive production of data [7].

Lack of standardised data production processes, fragmentation of databases, and errors and duplication in data production are only some of the challenges of RHISs, which may, at first glance be categorised as technical challenges $[3,8]$. Solutions to such apparently technical challenges include introducing new data forms, setting up warning systems to detect potential errors, and developing algorithms for integrating different databases.

However, DH interventions for RHISs may not be used effectively if data production and utilisation processes are viewed as merely technical. Given that RHISs are embedded in complex health systems, DH interventions to improve RHIS functions are also influenced by the broader setting, in which dynamic and complex social and technical factors interact [9-11]. There is a need to consider the influence of social factors as well. These may include people's competencies in dealing with new data production processes, institutional values about data utilisation, and existing relationships between data producers and decision-makers $[8,12,13]$.

There is growing recognition that the development and implementation of DH interventions occurs in multiple technical and social contexts, and that DH interventions may differ in definition, purpose and intended outcomes [14]. So, various terms are used for interventions with similar aims and activities to data harmonisation. For example, terms such as record linkage, data warehousing, data sharing and health information exchange are all used to describe data harmonisationtype activities [15-17]; and it is not always clear to which extent these efforts are similar in practice, scope and relevance. The use of multiple terms may not be a problem in itself, but a common understanding of the components and processes will bring more clarity about what constitutes 'data harmonisation', and will make it easier to compare and appraise the relevance and usefulness of DH interventions across settings.

Although DH has the potential to enhance RHISs, it is still unclear whether or how it affects health management decision-making. In some cases, $\mathrm{DH}$ interventions may not directly impact on improved management decision-making, especially when interventions are more focused on the technical aspects of data production and less on the organisational and behavioural aspects of data use for decision-making [18]. The scope of this review is to therefore understand the different ways in which $\mathrm{DH}$ is defined, to identify its components and processes, and to describe whether or how DH can affect health management decision-making. Greater clarity about the range of definitions, components and processes of DH interventions, and its intended outcomes can help to better evaluate its relevance, usefulness, and impact [12].

\section{Methods}

This scoping review was conducted according to the methods outlined by Arksey and O'Malley [19]. They recommend a process that is "not linear but, requiring researchers to engage with each stage in a reflexive way" to achieve both 'in-depth and broad' results. This review followed the standard steps for systematic reviews: identifying the research question, identifying relevant studies, selecting studies for inclusion, data extraction and data synthesis. These are detailed in our published study protocol [20].

\section{Study objectives}

This scoping review appraised the definitions, components and processes of data harmonisation activities, and provided a broad explanation of the relationship between data harmonisation interventions and health management decision-making. The specific objectives are:

1. To identify and synthesise the various definitions, components and processes of data harmonisation in healthcare; and

2. To describe the relationship between data harmonisation interventions and health management decision-making.

We took a stepped approach in addressing these objectives. All included studies were used to address 
Objective 1. To address Objective 2, we sampled studies that were using alternative terms for $\mathrm{DH}$ interventions and used those to identify, synthesise and compare similarities and differences in definitions. While executing Objective 1 and 2, we identified a smaller number of studies that contributed to Objective 3 .

\section{Identifying relevant studies Eligibility criteria}

Peer-reviewed studies and grey literature were considered eligible for inclusion into the scoping review if they provided a definition or description of $\mathrm{DH}$, and or, a more detailed conceptual explanation (in the form of a model, framework or process) of a $\mathrm{DH}$ intervention. Additionally, studies were eligible if they provided an explanation of the causal relationship between $\mathrm{DH}$ and health management decision-making (such as through improved quality and accessibility of harmonised information for management and/or the utilisation of harmonised health information for management decisionmaking). We considered any studies concerned with different technical activities of DH (such as linking, merging, cleaning and transferring). After screening, only studies for which we could access full-text articles were eligible for inclusion in the review.

\section{Search strategy}

A systematic literature search was conducted in PubMed, CINAHL and Web of Science for eligible studies from 1 January 2000 to 30 September 2018. We limited our search to the year 2000 as digital technologybased innovations began during this period (such as health information exchange) began in high-income countries (predominantly in the United States of America) and when researchers and health system managers in LMICs became interested in the integration of large digital databases [21]. We present the search strategy in the study protocol [20]. Based on preliminary searches we anticipated that these databases would yield the highest results. The search strategies include a combination of keywords and Medical Subject Headings (MeSH) terms related to data harmonisation (concept A) and health information system (concept B). There were no geographic restrictions, but for logistical reasons of time and resources, we only searched for English studies.

\section{Selecting studies for inclusion Screening records}

The first reviewer (BS) conducted all the searches with the help of a librarian and collated the records in the EndNote reference management programme where duplicates were removed. Two reviewers (BS) and second reviewer $(\mathrm{AH})$ then independently screened the records (titles and abstracts) to assess eligibility for full-text review. $\mathrm{BS}$ and $\mathrm{AH}$ resolved conflicts that emerged at this stage by talking through the inclusion criteria and arriving at a joint decision.

The full-texts of potentially eligible studies were retrieved and assessed by the two reviewers (BS and $\mathrm{AH}$ ). Final inclusion into the review was based on whether at a minimum the study had a definition or description of a DH intervention or referred to its relationship with health management decision-making. The first reviewer read all full-texts and the second reviewer only read a sample (roughly a third) of the full-texts to verify the first reviewer's decision about inclusion. BS and AH disagreed on four studies, and after discussion, agreed to exclude the studies.

After finalising screening, the two reviewers then mapped out the characteristics of included studies in an Excel spreadsheet. They recorded the name of the first author, the date, the type of study (primary, review, conceptual, commentary), the term used for the intervention they described (DH or alternative), the country in which the study was taking place, level at which the intervention was implemented (frontline, management, research), and ticked whether there was a conceptual model, framework, diagram or process description of $\mathrm{DH}$ and health management decision-making. This detailed mapping of study characteristics was useful for informing sampling options for Objectives 2 and 3.

\section{Sampling of studies}

A scoping review aims to map the literature on a particular topic rather than to provide an exhaustive explanation of a particular phenomenon of interest $[19,22]$. Thus, the number of included studies was expected to be high in the scoping reviews. To manage the high numbers for a scoping review such as this one (where the aim was to provide definitions and concepts) it was necessary to make use of a qualitative sampling approach. A qualitative sampling approach for this review aimed for variation and depth rather than an exhaustive sample; reviewing too large a number of studies can impair the quality of the analysis and synthesis [23]. We used two types of purposive sampling techniques called maximum variation sampling and theoretical sampling [24]. These techniques were used to identify both the range, variation and similarities or differences in definitions and concepts and intervention descriptions (as per Objective 2) and to provide a rich synthesis of explanations of causal relationships between $\mathrm{DH}$ and health management decision-making (as per Objective 3). For Objective 1, we did not apply a sampling strategy. Thus, we included all the studies that at a minimum provided a definition or description of a DH intervention.

\section{Data extraction}

BS extracted data for Objective 1 from all the included studies $(n=181)$. AH independently extracted data from 
$81(45 \%)$ of included studies to verify data extraction done by the first reviewer. We used an MS Excel spreadsheet for data extraction as presented in Fig. 1. $\mathrm{AH}$ and $\mathrm{BS}$ extracted a few studies before clarifying the items in the spreadsheet. Once data extraction was complete, the reviewers were able to filter according to the individual items extracted to synthesise and compare studies. Given the objectives of the scoping review, we did not extract any information relevant for conducting risk of bias or quality assessment. Not conducting risk of bias or quality assessment is consistent with scoping reviews of similar aims and methodological approaches [19, 22, 25].

\section{Data synthesis: collating, summarising and reporting findings}

The first reviewer (BS) conducted data analysis using manual coding and the filter option in MS Excel. Another reviewer (NL) reviewed the data analysis work on an ongoing basis as an additional quality check. For Objective 1 , we conducted a numerical analysis to provide an overview of the characteristics of all the included studies. For Objective 2, we conducted a qualitative analysis to provide a narrative synthesis of the different $\mathrm{DH}$ definitions and concepts, and to identify different components or activities that are considered part of the $\mathrm{DH}$ processes. For Objective 3, we reviewed data related to intentions, suggestions and or explanations of how DH may lead to improved health management decisionmaking. We extracted and analysed data relevant to Objective 2 and 3 at the same time. We first created a list of all the different terms used to describe DH interventions and then compared definitions across alternative terms by looking for similarities or differences in the definitions or descriptions of DH interventions. We then coded key components, processes and outcomes of DH interventions and the factors reported as important in the relationship between $\mathrm{DH}$ and health management decision-making.

The findings are structured according to three themes matching the three study objectives: an overview of the key characteristics of included studies, alternative terms and definitions of $\mathrm{DH}$, and a narrative synthesis of the relationship between $\mathrm{DH}$ and health management decision-making.

\section{Reflexivity}

Throughout the review, the authors were aware of their own positions and reflected on how these could influence the study design, search strategy, inclusion decisions, data extraction, analysis, and synthesis, and interpretation of the findings [23]. The review authors are trained in anthropology, epidemiology, health systems, and evidence synthesis research. The first author was involved in participant observation of an innovative DH project in the Western Cape Department of Health in South Africa as part of her doctoral research where she grappled with questions that informed the objectives of this review. Three of the authors (BS, $\mathrm{AH}$ and $\mathrm{NL}$ ) were involved in a Cochrane systematic review on RHIS interventions when this scoping review was conceptualised, so they were familiar with some of the health information literature (HIS) literature and had some appreciation for the conceptual and methodological complexities of studying the field of health information management. This experience informed the way the first author developed the search strategy. She used an iterative approach to narrow down the search as much as possible because of her prior knowledge that it was difficult to balance sensitivity and specificity when developing a search strategy for HIS literature that is often multi-disciplinary in nature.

\section{Results}

\section{Results of the search}

Figure 2 shows a PRISMA diagram of the search results. We screened a total of 1331 records; 1232 titles and abstracts identified from searching three electronic databases, and 99 from screening for a Cochrane systematic review assessing the effectiveness of RHIS interventions on health systems management [26] and grey literature. Almost a quarter (289 of 1331) were deemed potentially eligible for full-text screening. We accessed full-texts for 275 studies and of those, 181 were included in the scoping review for Objective 1. We excluded 94 full-text articles because they

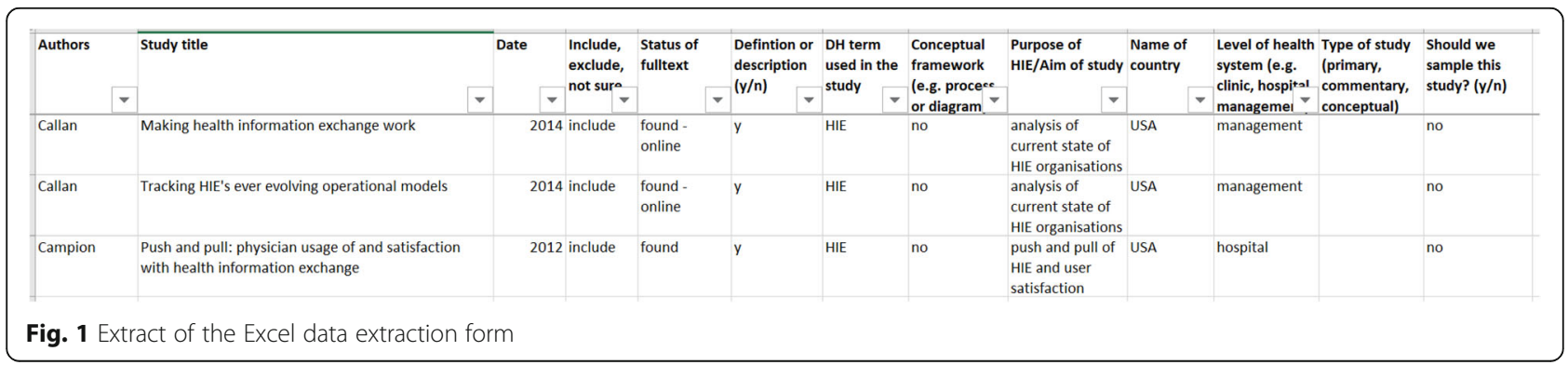


did not meet the minimum criteria; that is, provide a definition or description of a $\mathrm{DH}$ intervention or activity. We sampled 61 studies from the 181 for Objective 2 and 3 . We arrived at 61 studies by including all reviews (systematic or literature reviews) and all studies (irrespective of the type of study), that also had a process description, conceptual framework or theory of a DH intervention (that is, in addition to the minimum criteria for Objective 1).

\section{An overview of key characteristics of data harmonisation studies}

A total of 181 studies were included into this scoping review for Objective 1 (see Table 1). Given the high number of included studies, we decided to only map the following key characteristics of those studies: first author, date, type of study, intervention term ( $\mathrm{DH}$ or alternative), country and level of the health care system. Most included studies (126 of 181) were primary studies assessing various aspects of developing and implementing $\mathrm{DH}$ interventions (quantitative studies $n=86$ ) or patient, providers or stakeholders' perspectives (qualitative studies $n=34$ ) or a combination of both (mixed methods studies $n=6$ ).

Of the 181 included studies, 9 were not country specific (these were global reviews), 151 were from the USA and the rest were from other countries (specifically Australia, Brazil, Canada, China, Finland, Germany, Israel, Japan, Jordan, Korea, Malaysia, Netherlands, South Africa and South Korea). In terms of the level of the health care system, 128 studies were on a DH intervention or activity that was concerned with the frontline level (health service providers), 48 studies were concerned with health system factors or policy-related activities at the managerial level, and 5 studies focused on $\mathrm{DH}$ interventions specifically for research purposes. Most studies (92\%) used the term health information exchange (HIE), while the remaining studies (8\%) used a variety of terms to describe various $\mathrm{DH}$ interventions and activities, specifically, record linkage, data mining, data linkage, data warehousing, data sharing and data harmonisation.

\section{Definitions, components and processes of data harmonisation}

We first discuss the alternative terms and definitions of $\mathrm{DH}$ and then we summarise key components and processes of DH using studies sampled from the 61 studies identified for Objective 2 and 3. Table 2 presents identifying details of the 61 studies; that is, the type of study design, the intervention terms, the country, the level of

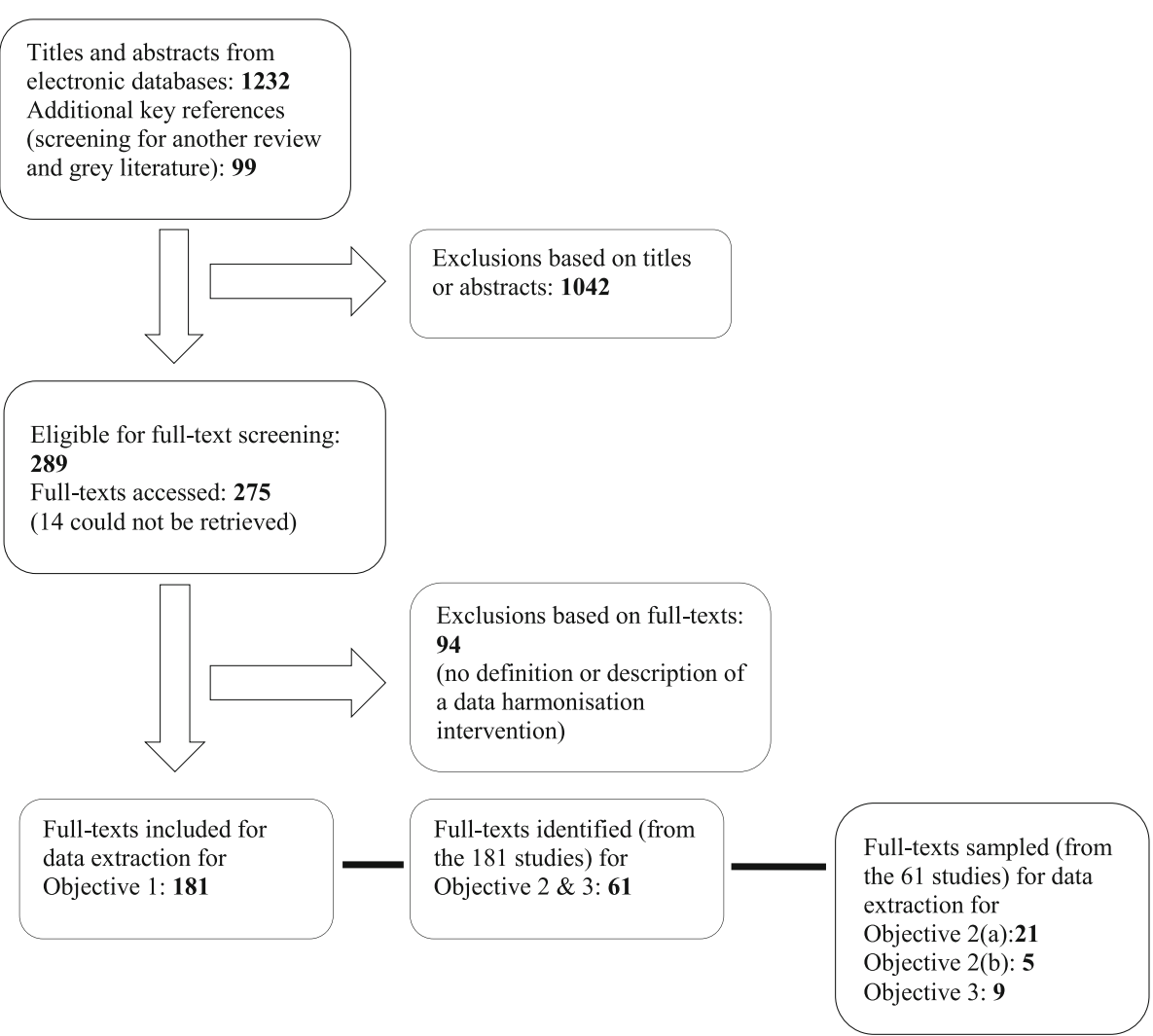

Fig. 2 PRISMA diagram of eligible studies 
Table 1 Characteristics of included studies $(n=181)$

\begin{tabular}{|c|c|c|c|c|c|}
\hline Study name & Date & Type of study & Intervention term & Country & Level of the health care system \\
\hline \multicolumn{6}{|l|}{ Commentary } \\
\hline Burris & 2017 & Commentary & HIE & USA & Frontline: hospitals \\
\hline Figge & 2010 & Commentary & HIE & USA & Management \\
\hline Mcllwain & 2009 & Commentary & HIE & USA & Management \\
\hline Murphy & 2010 & Commentary & HIE & USA & Management \\
\hline Overhage & 2007 & Commentary & HIE & USA & Management \\
\hline Rudin & 2010 & Commentary & HIE & USA & Frontline: workers \\
\hline \multicolumn{6}{|l|}{ Conceptual } \\
\hline Boyd & 2014 & Conceptual & $\mathrm{RL}$ & Australia & Research \\
\hline Carr & 2013 & Conceptual & HIE & USA & Frontline: hospitals \\
\hline Cimino & 2014 & Conceptual & HIE & USA & Management \\
\hline Deas & 2012 & Conceptual & HIE & USA & Management \\
\hline Del Fiol & 2015 & Conceptual & HIE & USA & Frontline: prisons, hospitals \\
\hline Dimitropoulos & 2009 & Conceptual & HIE & USA & Management \\
\hline Downs & 2010 & Conceptual & HIE & USA & Management \\
\hline Feldman & 2017 & Conceptual & HIE & USA & Management \\
\hline Frisse & 2010 & Conceptual & HIE & USA & Frontline: patients, workers \\
\hline Frisse & 2008 & Conceptual & HIE & USA & Frontline: organisations \\
\hline Frohlich & 2007 & Conceptual & HIE & USA & Management \\
\hline Godlove & 2015 & Conceptual & HIE & USA & Frontline: patients \\
\hline Greene & 2016 & Conceptual & HIE & USA & Management \\
\hline Grossman & 2008 & Conceptual & HIE & USA & Management \\
\hline Haarbrandt & 2016 & Conceptual & DW & USA & Management \\
\hline $\mathrm{Hu}$ & 2007 & Conceptual & DS & USA & Management \\
\hline Jones & 2012 & Conceptual & DS & USA & Management \\
\hline Kuperman & 2013 & Conceptual & HIE & USA & Management \\
\hline Langabeer & 2016 & Conceptual & HIE & USA & Management \\
\hline Liu & 2011 & Conceptual & HIE & China & Management \\
\hline McDonald & 2009 & Conceptual & HIE & USA & Management \\
\hline McMurray & 2015 & Conceptual & HIE & USA & Management \\
\hline Miller & 2014 & Conceptual & HIE & USA & Frontline: hospitals \\
\hline Nelson & 2016 & Conceptual & HIE & USA & Frontline: prisons, hospitals \\
\hline Politi & 2014 & Conceptual & HIE & $\mathrm{n} / \mathrm{a}$ & Management \\
\hline Ranade-Kharkar & 2014 & Conceptual & HIE & USA & Management \\
\hline Shapiro & 2016 & Conceptual & HIE & USA & Frontline: workers, organisations \\
\hline Shapiro & 2006 & Conceptual & HIE & USA & Management \\
\hline Thorn & 2013 & Conceptual & HIE & USA & Frontline: health care workers \\
\hline Thorn & 2014 & Conceptual & HIE & USA & Frontline: health care workers \\
\hline Vest & 2010 & Conceptual & HIE & USA & Management \\
\hline Williams & 2012 & Conceptual & HIE & USA & Management \\
\hline Yaraghi & 2014 & Conceptual & HIE & USA & Management \\
\hline Zafar & 2007 & Conceptual & HIE & USA & Management \\
\hline Zaidan & 2015 & Conceptual & HIE & Malaysia & Management \\
\hline
\end{tabular}


Table 1 Characteristics of included studies ( $n=181)$ (Continued)

\begin{tabular}{|c|c|c|c|c|c|}
\hline Study name & Date & Type of study & Intervention term & Country & Level of the health care system \\
\hline \multicolumn{6}{|c|}{ Primary studies } \\
\hline Abramson & 2012 & Primary, quantitative & EHR, HIE & USA & Frontline, hospitals \\
\hline Adjerid & 2011 & Primary, quantitative & HIE & USA & Management, states \\
\hline Adler-Milstein & 2011 & Primary, quantitative & HIE & USA & Frontline: organisations \\
\hline Adler-Milstein & 2013 & Primary, quantitative & HIE & USA & Management, organisations \\
\hline Adler-Milstein & 2016 & Primary, quantitative & HIE & USA & Management \\
\hline Alexander & 2016 & Primary, qualitative & HIE & USA & Frontline, health care workers \\
\hline Alexander & 2015 & Primary, qualitative & HIE & USA & Frontline, health care workers \\
\hline Ancker & 2012 & Primary, quantitative & HIE & USA & Frontline, consumers \\
\hline Bahous & 2016 & Primary, quantitative & HIE & Israel & Frontline, hospital \\
\hline Bailey & 2013 & Primary, quantitative & HIE & USA & Frontline: hospital \\
\hline Ben-Assuli & 2013 & Primary, quantitative & HIE & USA & Frontline: hospitals \\
\hline Boockvar & 2017 & Primary, quantitative & HIE & USA & Frontline: hospital \\
\hline Butler & 2014 & Primary, qualitative & HIE & USA & Frontline: prisons, communities \\
\hline Campion & 2012 & Primary, quantitative & HIE & USA & Frontline: health care workers \\
\hline Campion & 2013 & Primary, quantitative & HIE & USA & Frontline: communities \\
\hline Campion & 2013 & Primary, quantitative & HIE & USA & Frontline: clinics, hospitals \\
\hline Campion & 2014 & Primary, quantitative & DE & USA & Frontline: organisations \\
\hline Carr & 2014 & Primary, quantitative & HIE & USA & Frontline: hospitals \\
\hline Carr & 2016 & Primary, quantitative & HIE & USA & Frontline: hospitals \\
\hline Cochran & 2015 & Primary, qualitative & HIE & USA & Frontline: clinics, communities \\
\hline Cross & 2016 & Primary, qualitative & HIE & USA & Management, organisations \\
\hline Dalan & 2010 & Primary, qualitative & DM & USA & Research \\
\hline Dimitropoulos & 2011 & Primary, quantitative & HIE & USA & Frontline: consumers \\
\hline Dixon & 2013 & Primary, quantitative & HIE & USA & Frontline: hospitals \\
\hline Dixon & 2011 & Primary, quantitative & HIE & USA & Frontline: laboratories \\
\hline Downing & 2017 & Primary, quantitative & HIE & USA & Management: policy \\
\hline Dullabh & 2013 & Primary, qualitative & HIE & USA & Management: organisations \\
\hline Elysee & 2017 & Primary, quantitative & HIE, IO & USA & Frontline: hospitals \\
\hline Foldy & 2007 & Primary, quantitative & HIE & USA & Management: organisations \\
\hline Fontaine & 2010 & Primary, qualitative & HIE & USA & Frontline: primary health care \\
\hline French & 2016 & Primary, quantitative & HIE & USA & Management: organisations \\
\hline Fricton & 2008 & Primary, quantitative & HIE & USA & Frontline: patients, workers \\
\hline Frisse & 2012 & Primary, quantitative & HIE & USA & Frontline: organisations \\
\hline Furukawa & 2013 & Primary, quantitative & HIE & USA & Frontline: hospitals \\
\hline Furukawa & 2014 & Primary, quantitative & HIE & USA & Frontline: health care workers \\
\hline Gadd & 2011 & Primary, quantitative & HIE & USA & Frontline: health care workers \\
\hline Garg & 2014 & Primary, quantitative & HIE & USA & Frontline: hospitals \\
\hline Gill & 2001 & Primary, quantitative & $\mathrm{DL}$ & South Africa & Frontline: patients, disease \\
\hline Grinspan & 2013 & Primary, quantitative & HIE & USA & Frontline: patients \\
\hline Grinspan & 2014 & Primary, quantitative & HIE & USA & Frontline: health care workers \\
\hline Grinspan & 2015 & Primary, quantitative & HIE & USA & Frontline: patients \\
\hline Hassol & 2014 & Primary, quantitative & HIE & USA & Frontline: health care workers \\
\hline Herwehe & 2012 & Primary, quantitative & HIE & USA & Frontline: health care workers \\
\hline
\end{tabular}


Table 1 Characteristics of included studies ( $n=181)$ (Continued)

\begin{tabular}{|c|c|c|c|c|c|}
\hline Study name & Date & Type of study & Intervention term & Country & Level of the health care system \\
\hline Hincapie & 2011 & Primary, qualitative & HIE & USA & Frontline: health care workers \\
\hline Holman & 2008 & Primary, quantitative & $\mathrm{DL}$ & USA & Frontline: organisations, research \\
\hline Hypponen & 2014 & Primary, quantitative & HIE & Finland & Frontline: health care workers \\
\hline $\mathrm{Ji}$ & 2017 & Primary, quantitative & HIE & Korea & Frontline: hospitals \\
\hline Johnson & 2011 & Primary, mixed & HIE & USA & Frontline: hospitals \\
\hline Jung & 2015 & Primary, quantitative & HIE & USA & Frontline: hospitals \\
\hline Kaelber & 2013 & Primary, quantitative & HIE & USA & Frontline: hospitals \\
\hline Kierkegaard & 2014 & Primary, qualitative & HIE & USA & Frontline: health care workers \\
\hline Kierkegaard & 2014 & Primary, qualitative & HIE & USA & Management \\
\hline Kim & 2012 & Primary, qualitative & HIE & Korea & Management \\
\hline Knaup & 2006 & Primary. quantitative & DS & Germany & Frontline: hospitals \\
\hline Kralewski & 2012 & Primary, qualitative & CIE & USA & Frontline: organisations, workers \\
\hline Laborde & 2011 & Primary, quantitative & HIE & USA & Frontline: hospitals \\
\hline Lee & 2012 & Primary, quantitative & HIE & South Korea & Frontline: health care workers \\
\hline $\mathrm{Li}$ & 2011 & Primary, quantitative & DE & Japan \& China & Frontline: organisations \\
\hline Liu & 2010 & Primary, qualitative & $\mathrm{DH}$ & China & Management \\
\hline Lobach & 2007 & Primary, quantitative & HIE & USA & Management \\
\hline Maenpaa & 2011 & Primary, quantitative & HIE & Finland & Frontline: hospital \\
\hline Maiorana & 2012 & Primary, mixed & HIE & USA & Frontline: workers, disease \\
\hline Martinez & 2015 & Primary, quantitative & HIE & USA & Frontline: hospitals \\
\hline Massoudi & 2016 & Primary, qualitative & HIE & USA & Frontline: organisations \\
\hline Mastebroek & 2017 & Primary, qualitative & HIE & Netherlands & Frontline: patients \\
\hline Mastebroek & 2017 & Primary, qualitative & HIE & Netherlands & Frontline: patients \\
\hline Mastebroek & 2016 & Primary, quantitative & HIE & Netherlands & Frontline: health care workers \\
\hline Matsumoto & 2017 & Primary, qualitative & $\mathrm{HIE}$ & USA & Frontline: workers, hospitals \\
\hline Medford-Davis & 2017 & Primary, quantitative & HIE & USA & Frontline: patients, hospitals \\
\hline Mello & 2018 & Primary, qualitative & HIE & USA & Management: policies \\
\hline Merrill & 2013 & Primary, quantitative & HIE & USA & Frontline: managers \\
\hline Messer & 2012 & Primary, mixed & HIE & USA & Frontline: clinics, organisations \\
\hline Miller & 2012 & Primary, qualitative & HIE & USA & Frontline: consumers, organisations \\
\hline Miller & 2017 & Primary, quantitative & HIE & USA & Frontline: disease, workers \\
\hline Moore & 2012 & Primary, quantitative & HIE & USA & Frontline: workers, hospitals \\
\hline Motulsky & 2018 & Primary, quantitative & HIE & Canada & Frontline: workers \\
\hline Myers & 2012 & Primary, qualitative & HIE & USA & Frontline: disease, workers \\
\hline Obeidat & 2014 & Primary, quantitative & IE & Jordan & Frontline: hospitals \\
\hline O'Donnell & 2011 & Primary, quantitative & HIE & USA & Frontline: workers \\
\hline Onyile & 2013 & Primary, quantitative & HIE & USA & Frontline: patients \\
\hline Opoku-Agyeman & 2016 & Primary, quantitative & HIE & USA & Frontline: hospitals \\
\hline Overhage & 2017 & Primary, quantitative & HIE & USA & Management \\
\hline Ozkaynak & 2013 & Primary, qualitative & HIE & USA & Frontline: hospitals, workers \\
\hline Park & 2015 & Primary, quantitative & HIE & South Korea & Frontline: clinics, hospitals \\
\hline Park & 2013 & Primary, quantitative & HIE & South Korea & Frontline: clinics, hospitals \\
\hline Patel & 2011 & Primary, quantitative & HIE & USA & Frontline: clinics, hospitals \\
\hline Politi & 2015 & Primary, quantitative & HIE & Israel & Frontline: hospital \\
\hline
\end{tabular}


Table 1 Characteristics of included studies ( $n=181)$ (Continued)

\begin{tabular}{|c|c|c|c|c|c|}
\hline Study name & Date & Type of study & Intervention term & Country & Level of the health care system \\
\hline Ramos & 2016 & Primary, mixed & HIE & USA & Frontline: patients \\
\hline Ramos & 2014 & Primary, qualitative & HIE & USA & Frontline: patients \\
\hline Reis & 2016 & Primary, quantitative & $\mathrm{HDE}$ & USA & Frontline: hospital \\
\hline Richardson & 2014 & Primary, qualitative & HIE & USA & Frontline: organisations, workers \\
\hline Ross & 2010 & Primary, qualitative & HIE & USA & Frontline: clinics \\
\hline Ross & 2013 & Primary, quantitative & HIE & USA & Frontline: workers, clinics, hospitals \\
\hline Rudin & 2009 & Primary, qualitative & HIE & USA & Frontline: health care workers \\
\hline Rundall & 2016 & Primary, qualitative & HIE & USA & Management: policy makers, leaders \\
\hline Saef & 2014 & Primary, quantitative & HIE & USA & Frontline: hospitals \\
\hline Santos & 2017 & Primary, quantitative & HIE & Brazil & Frontline: clinics, hospitals \\
\hline Shade & 2012 & Primary, quantitative & HIE & USA & Frontline: clinics, hospitals \\
\hline Shank & 2012 & Primary, quantitative & $\mathrm{HIE}$ & USA & Frontline: health care workers \\
\hline Shapiro & 2013 & Primary, quantitative & HIE & USA & Frontline: hospitals \\
\hline Shapiro & 2007 & Primary, quantitative & HIE & USA & Frontline: health care workers \\
\hline Sicotte & 2010 & Primary, qualitative & HIE & Canada & Frontline: workers, hospitals \\
\hline Sprivulis & 2007 & Primary, quantitative & HIE & Australia & Frontline: workers, organisations \\
\hline Squire & 2002 & Primary, mixed & HIE & USA & Frontline: health care workers \\
\hline Sridhar & 2012 & Primary, quantitative & HIE & USA & Frontline: hospital \\
\hline Thornewill & 2011 & Primary, mixed & HIE & USA & Frontline: consumers, organisations \\
\hline Unertl & 2012 & Primary, qualitative & HIE & USA & Frontline: clinics, hospitals \\
\hline Vest & 2010 & Primary, qualitative & HIE & USA & Frontline: hospitals \\
\hline Vest & 2017 & Primary, qualitative & HIE & USA & Frontline: consumers, organisations \\
\hline Vest & 2015 & Primary, qualitative & HIE & USA & Frontline: consumers, organisations \\
\hline Vest & 2013 & Primary, qualitative & HIE & USA & Management: policy makers \\
\hline Vest & 2009 & Primary, quantitative & HIE & & Frontline: workers, patients \\
\hline Vest & 2017 & Primary, quantitative & $\mathrm{HIE}$ & USA & Frontline: consumers, organisations \\
\hline Vest & 2011 & Primary, quantitative & HIE & USA & Frontline: patients, hospitals \\
\hline Vest & 2014 & Primary, quantitative & HIE & USA & Frontline: patients, hospitals \\
\hline Vest & 2014 & Primary, quantitative & HIE & USA & Frontline: hospitals \\
\hline Vest & 2015 & Primary, quantitative & HIE & USA & Frontline: hospitals \\
\hline Vreeman & 2008 & Primary, quantitative & HIE & USA & Frontline: laboratory, radiology \\
\hline Wen & 2010 & Primary, quantitative & HIE & USA & Frontline: patients \\
\hline Winden & 2014 & Primary, quantitative & HIE & USA & Frontline: clinical care \\
\hline Wright & 2010 & Primary, quantitative & HIE & USA & Frontline: health care workers \\
\hline Yeager & 2014 & Primary, qualitative & HIE & USA & Frontline: consumers \\
\hline Yeaman & 2015 & Primary, quantitative & HIE & USA & Frontline: hospital \\
\hline Zech & 2015 & Primary, quantitative & $\mathrm{HIE}$ & USA & Frontline: patients, organisations \\
\hline Zech & 2016 & Primary, quantitative & HIE & USA & Frontline: patients, organisations \\
\hline Zhu & 2010 & Primary quantitative & $\mathrm{HIE}$ & USA & Research \\
\hline \multicolumn{6}{|l|}{ Study protocol } \\
\hline Dixon & 2013 & Protocol, mixed & HIE & USA & Frontline: organisations \\
\hline \multicolumn{6}{|l|}{ Reviews } \\
\hline Esmaeilzadeh & 2016 & Review & HIE & $\mathrm{n} / \mathrm{a}$ & Management: policy \\
\hline Esmaeilzadeh & 2017 & Review & HIE & $\mathrm{n} / \mathrm{a}$ & Frontline: patients \\
\hline
\end{tabular}


Table 1 Characteristics of included studies ( $n=181)$ (Continued)

\begin{tabular}{|c|c|c|c|c|c|}
\hline Study name & Date & Type of study & Intervention term & Country & Level of the health care system \\
\hline Fontaine & 2010 & Review & HIE & $\mathrm{n} / \mathrm{a}$ & Frontline: primary health care \\
\hline Hopf & 2014 & Review & $\mathrm{DL}$ & $\mathrm{n} / \mathrm{a}$ & Frontline: health care workers \\
\hline Kash & 2017 & Review & HIE & $\mathrm{n} / \mathrm{a}$ & Frontline: hospitals \\
\hline Mastebroek & 2014 & Review & $\mathrm{HIE}$ & USA & Frontline: disease, workers \\
\hline Parker & 2016 & Review & HIE & USA & Research \\
\hline Rahurkar & 2015 & Review & $\mathrm{HIE}$ & $\mathrm{n} / \mathrm{a}$ & Frontline: hospital \\
\hline Rudin & 2014 & Review & HIE & USA & Frontline: clinical care \\
\hline Sadoughi & 2018 & Review & $\mathrm{HIE}$ & $\mathrm{n} / \mathrm{a}$ & Management \\
\hline Vest & 2012 & Review & HIE & $\mathrm{n} / \mathrm{a}$ & Management \\
\hline Dixon & 2010 & Review & HIE & USA & Research \\
\hline Akhlaq & 2016 & Review & HIE & LMICs & Management, countries \\
\hline
\end{tabular}

the health care system and the purpose of the study (see Table 2). These studies were concerned with the challenges and opportunities of $\mathrm{DH}$, the barriers and facilitators of $\mathrm{DH}$, the various factors affecting $\mathrm{DH}$ (such as technical and financial factors), the outcomes of $\mathrm{DH}$ (such as patient safety and quality of care), and privacy and security issues of patient information.

\section{Alternative terms and definitions of data harmonisation}

For Objective 2 (a), we describe alternative terms and definitions of DH. We sampled 21 studies from the 61 studies identified for Objective 2 and 3. The alternative terms and definitions are summarised in Table 3. During data analysis we realised that most studies (53 of 61) used term 'health information exchange', with similar definitions. We sample 13 of the 53 studies to contribute to the composite HIE definition in the table. These 13 studies were chosen to represent the term HIE because they were review studies and we assumed that reviews provided synthesised definitions of interventions. Using maximum variation sampling, we included 8 more studies (21 studies in total), because they provided a range of different terms for DH activities, besides the term HIE.

There is overlap between the terms and definitions. Definitions for data harmonisation, record linkage and data warehousing explicitly state that these interventions involve a process of having to integrate different or 'homogeneous' databases or information systems. Data linkage and record linkage both focus on 'linkage' as a core activity in combining different databases using a unique patient identifier. HIE is described as a key outcome of data interoperability, that is, where the focus is on technical linkage of different electronic data bases. Data sharing, where the focus is on data accessibility and use, is described as a key outcome of HIE.

Based on the literature, we identified elements found in the various definitions of data harmonisation. DH is considered a multi-step process with a range of activities (such as identifying, reviewing, matching, redefining and standardising information). Data harmonisation interventions rely on interoperability between databases and systems which means copying standardised patient-level data into a separate repository. Data linkage and record linkage are activities of a broader intervention (data harmonisation), using mechanisms (such as unique patient identifiers) for integrating large datasets. Data warehousing is concerned with extracting, transforming and loading large datasets using information technology (IT) platforms, application systems and data displays (data marts or data dashboards). Data sharing (through the accessing and exchanging electronic health information), can be considered an outcome of HIE interventions. The aim of these interventions is to integrate and make data accessible across different platforms (such as clinical and financial systems), and to allow for the sharing of this data across the patient care trajectory. The ultimate aim of $\mathrm{DH}$, it would seem, is to improve patient outcomes, coordination of health services, quality of care and efficiency and facilitate public health interventions.

In reviewing the definitions, we identified nine characteristics of DH. No single study included all these characteristics, and there are no specific factors such as study design, country or level of the health care system associated with the definitions. DH is characterised by the following characteristics:

- Any type of DH intervention or activity is a process of multiple steps involving both technical and social processes.

- The goal of a DH intervention or activity is to integrate, harmonise and bring together different electronic databases into useable formats.

- There are at least two or more databases involved in any DH intervention or activity.

- A data harmonisation intervention or activity involves electronic data (no reference is made to data found in paper-based sources). 
Table 2 Characteristics of sampled studies $(n=61)$

\begin{tabular}{|c|c|c|c|c|c|c|}
\hline Study name & Date & Type of study & $\begin{array}{l}\text { Intervention } \\
\text { term }\end{array}$ & Country & $\begin{array}{l}\text { Level of the health } \\
\text { care system }\end{array}$ & Purpose of the study \\
\hline Akhlaq & 2016 & Review, qualitative & HIE & LMICs & $\begin{array}{l}\text { Management, } \\
\text { countries }\end{array}$ & Barriers and facilitators of HIE \\
\hline Boyd Boyd & 2014 & Conceptual & $\mathrm{RL}$ & Australia & Research & Functions of record linkage \\
\hline Burris & 2017 & Commentary & HIE & USA & Frontline: hospitals & Benefits of HIE \\
\hline Campion & 2012 & Primary, quantitative & HIE & USA & $\begin{array}{l}\text { Frontline: health } \\
\text { care workers }\end{array}$ & Push and pull of HIE \\
\hline Cimino & 2014 & Conceptual & HIE & USA & Management & Debates around consumer-mediated HIE \\
\hline Dalan & 2010 & Conceptual & DM & USA & Management & Possibilities for clinical data mining and research \\
\hline Dimitropoulos & 2009 & Conceptual & HIE & USA & Management & Privacy and security of interoperable HIE \\
\hline Dixon & 2010 & Review, framework & HIE & USA & Research & Costs, effort and value of HIE \\
\hline Downing & 2017 & Primary, quantitative & HIE & USA & Management: policy & $\begin{array}{l}\text { Relationship between HIE and organisational } \\
\text { HIE policy decisions }\end{array}$ \\
\hline Downs & 2010 & Conceptual & HIE & USA & Management & Improving laboratory services through HIE \\
\hline Dullabh & 2013 & Primary, qualitative & HIE & USA & $\begin{array}{l}\text { Management: } \\
\text { organisations }\end{array}$ & Experience of HIE implementation \\
\hline Elysee Elysee & 2017 & Primary, quantitative & $\mathrm{HIE}, \mathrm{IO}$ & USA & Frontline: hospitals & $\begin{array}{l}\text { Relationship between HIE, interoperability and } \\
\text { medication reconciliation }\end{array}$ \\
\hline Esmaeilzadeh & 2016 & Review & HIE & $\mathrm{n} / \mathrm{a}$ & Management: policy & HIE assimilation and patterns for policy \\
\hline Esmaeilzadeh & 2017 & Review & HIE & $\mathrm{n} / \mathrm{a}$ & Frontline: patients & Patients' perceptions of HIE \\
\hline Fontaine & 2010 & Review & HIE & $\mathrm{n} / \mathrm{a}$ & $\begin{array}{l}\text { Frontline: primary } \\
\text { health care }\end{array}$ & HIE for primary health care practices \\
\hline Fontaine & 2010 & Primary, qualitative & HIE & USA & $\begin{array}{l}\text { Frontline: primary } \\
\text { health care }\end{array}$ & $\begin{array}{l}\text { Barriers and facilitators of HIE in primary } \\
\text { care practices }\end{array}$ \\
\hline Frisse & 2010 & Conceptual & HIE & USA & $\begin{array}{l}\text { Frontline: patients, } \\
\text { workers }\end{array}$ & Impact of HIE on patient-provider relationships \\
\hline Gadd & 2011 & Primary, quantitative & HIE & USA & $\begin{array}{l}\text { Frontline: health } \\
\text { care workers }\end{array}$ & $\begin{array}{l}\text { Users' perspectives on the usability of a } \\
\text { regional HIE }\end{array}$ \\
\hline Gill & 2001 & Primary, quantitative & $\mathrm{DL}$ & South Africa & $\begin{array}{l}\text { Frontline: patients, } \\
\text { disease }\end{array}$ & Linkage of non-communicable diseases data \\
\hline Greene & 2016 & Conceptual & HIE & USA & Management & Technical and financial aspects of HIE \\
\hline Grossman & 2008 & Conceptual & HIE & USA & Management & Barriers to stakeholder participation in HIE \\
\hline Haarbrandt & 2016 & Conceptual & DW & USA & Management & Approaches for a clinical data warehouse \\
\hline Herwehe & 2012 & Primary, quantitative & HIE & USA & $\begin{array}{l}\text { Frontline: health } \\
\text { care workers }\end{array}$ & $\begin{array}{l}\text { Implementation of an electronic medical } \\
\text { record and HIE }\end{array}$ \\
\hline Hincapie & 2011 & Primary, qualitative & HIE & USA & $\begin{array}{l}\text { Frontline: health } \\
\text { care workers }\end{array}$ & Physicians' opinions of HIE \\
\hline Hopf & 2014 & Review & $\mathrm{DL}$ & $\mathrm{n} / \mathrm{a}$ & $\begin{array}{l}\text { Frontline: health } \\
\text { care workers }\end{array}$ & $\begin{array}{l}\text { Healthcare professionals' views of linking } \\
\text { routinely collected data }\end{array}$ \\
\hline $\mathrm{Hu}$ & 2007 & Conceptual & DS & USA & Management & $\begin{array}{l}\text { Challenges in implementing an infectious } \\
\text { disease information sharing and analysis } \\
\text { system }\end{array}$ \\
\hline Hypponen & 2014 & Primary, quantitative & HIE & Finland & $\begin{array}{l}\text { Frontline: health } \\
\text { care workers }\end{array}$ & User experiences with different regional HIE \\
\hline $\mathrm{Ji}$ & 2017 & Primary, quantitative & HIE & Korea & $\begin{array}{l}\text { Frontline: } \\
\text { hospitals }\end{array}$ & Technology and policy changes for HIE \\
\hline Jones & 2012 & Conceptual & DS & USA & Management & An overview of electronic data sharing \\
\hline Kash & 2017 & Review & HIE & $\mathrm{n} / \mathrm{a}$ & $\begin{array}{l}\text { Frontline: } \\
\text { hospitals }\end{array}$ & $\begin{array}{l}\text { Hospital readmission reduction and the role } \\
\text { of HIE }\end{array}$ \\
\hline Kierkegaard & 2014 & Primary, qualitative & HIE & USA & $\begin{array}{l}\text { Frontline: health } \\
\text { care workers }\end{array}$ & $\begin{array}{l}\text { Applications of HIE information to public } \\
\text { health practice }\end{array}$ \\
\hline
\end{tabular}


Table 2 Characteristics of sampled studies ( $n=61$ ) (Continued)

\begin{tabular}{|c|c|c|c|c|c|c|}
\hline Study name & Date & Type of study & $\begin{array}{l}\text { Intervention } \\
\text { term }\end{array}$ & Country & $\begin{array}{l}\text { Level of the health } \\
\text { care system }\end{array}$ & Purpose of the study \\
\hline Kierkegaard & 2014 & Primary, qualitative & HIE & USA & Management & Health practitioners' needs and HIE \\
\hline Kuperman & 2013 & Conceptual & HIE & USA & Management & Potential unintended consequences of HIE \\
\hline Liu & 2010 & Primary, qualitative & $\mathrm{DH}$ & China & Management & Defining data elements for HIE \\
\hline Maiorana & 2012 & Primary, mixed & HIE & USA & $\begin{array}{l}\text { Frontline: workers, } \\
\text { disease }\end{array}$ & $\begin{array}{l}\text { Trust, confidentiality and acceptability of } \\
\text { sharing HIV data for HIE }\end{array}$ \\
\hline Massoudi & 2016 & Primary, qualitative & HIE & USA & $\begin{array}{l}\text { Frontline: } \\
\text { organisations }\end{array}$ & HIE for clinical quality measures \\
\hline Mastebroek & 2014 & Review & HIE & USA & $\begin{array}{l}\text { Frontline: disease, } \\
\text { workers }\end{array}$ & $\begin{array}{l}\text { HIE in general care practice for people with } \\
\text { disabilities }\end{array}$ \\
\hline Mastebroek & 2016 & Primary, quantitative & HIE & Netherlands & $\begin{array}{l}\text { Frontline: health } \\
\text { care workers }\end{array}$ & Priority setting and feasibility of HIE \\
\hline Mastebroek & 2017 & Primary, qualitative & HIE & Netherlands & Frontline: patients & $\begin{array}{l}\text { Experiences of people with intellectual } \\
\text { disabilities in HIE }\end{array}$ \\
\hline Matsumoto & 2017 & Primary, qualitative & HIE & USA & $\begin{array}{l}\text { Frontline: workers, } \\
\text { hospitals }\end{array}$ & HIE in managing hospital services \\
\hline Parker & 2016 & Review & HIE & USA & Research & The use of HIE in supporting clinical research \\
\hline Politi & 2014 & Conceptual & HIE & n/a & Management & Use patterns of HIE \\
\hline Rahurkar & 2015 & Review & HIE & $\mathrm{n} / \mathrm{a}$ & Frontline: hospital & Impact of HIE on cost, use and quality of care \\
\hline Ramos & 2016 & Primary, mixed & HIE & USA & Frontline: patients & HIE consent process in an HIV clinic \\
\hline Ranade-Kharkar & 2014 & Conceptual & HIE & USA & Management & Improving data quality integrity through HIE \\
\hline Ross & 2010 & Primary, qualitative & HIE & USA & Frontline: clinics & $\begin{array}{l}\text { Motivators, barriers, and potential facilitators } \\
\text { of adoption of HIE }\end{array}$ \\
\hline Rudin & 2014 & Review & HIE & USA & $\begin{array}{l}\text { Frontline: clinical } \\
\text { care }\end{array}$ & Use and effect of HIE on clinical care \\
\hline Vest & 2016 & Primary, qualitative & HIE & USA & $\begin{array}{l}\text { Management: } \\
\text { policy makers, } \\
\text { leaders }\end{array}$ & Information-sharing needs and HIE \\
\hline Sadoughi & 2018 & Review & HIE & $\mathrm{n} / \mathrm{a}$ & Management & $\begin{array}{l}\text { Quality and cost-effectiveness, and the rates } \\
\text { of HIE adoption and participation }\end{array}$ \\
\hline Santos & 2017 & Primary, quantitative & HIE & Brazil & $\begin{array}{l}\text { Frontline: clinics, } \\
\text { hospitals }\end{array}$ & $\begin{array}{l}\text { HIE for continuity } \\
\text { of maternal and neonatal care }\end{array}$ \\
\hline Shade & 2012 & Primary, quantitative & HIE & USA & $\begin{array}{l}\text { Frontline: clinics, } \\
\text { hospitals }\end{array}$ & HIE for quality and continuity of HIV care \\
\hline Shapiro & 2016 & Conceptual & HIE & USA & $\begin{array}{l}\text { Frontline: workers, } \\
\text { organisations }\end{array}$ & HIE in emergency medicine \\
\hline Shapiro & 2006 & Conceptual & HIE & USA & Management & $\begin{array}{l}\text { Approaches to patient HIE and their impact on } \\
\text { emergency medicine }\end{array}$ \\
\hline Vest & 2012 & Review & HIE & $n / a$ & Management & $\begin{array}{l}\text { National and international approaches of health } \\
\text { information exchange }\end{array}$ \\
\hline Vest & 2015 & Primary, qualitative & HIE & USA & $\begin{array}{l}\text { Frontline: } \\
\text { consumers, } \\
\text { organisations }\end{array}$ & HIE to change cost and utilisation outcomes \\
\hline Vest & 2010 & Conceptual & HIE & USA & Management & Challenges and strategies for HIE \\
\hline Williams & 2012 & Conceptual & HIE & USA & Management & Strategies to advance HIE \\
\hline Yaraghi & 2014 & Conceptual & HIE & USA & Management & $\begin{array}{l}\text { Professional and geographical network effects } \\
\text { on HIE growth }\end{array}$ \\
\hline Yeager & 2014 & Primary, qualitative & HIE & USA & $\begin{array}{l}\text { Frontline: } \\
\text { consumers }\end{array}$ & Factors related to HIE participation and use \\
\hline Zaidan & 2015 & Conceptual & HIE & Malaysia & Management & Security framework for nationwide HIE \\
\hline Zhu & 2010 & Primary quantitative & HIE & USA & Research & Facilitating clinical research through HIE \\
\hline
\end{tabular}


Table 3 Alternative terms and definitions of data harmonisation interventions

Diu 2010 [1]
inhomogeneous information systems through identifying, reviewing, matching, redefining and standardising
information. This process involves two steps. Firstly, identifying whether all the information necessary for a
single electronic platform is available in existing systems, where the information is, and how the information
is defined and formatted. And secondly, to make the heterogeneous information recorded by various systems
consistent or at least comparable with one another by reviewing, matching, redefining and standardising each
data item.

Record linkage is the process of bringing together data relating to the same individual from within and between different datasets. When a unique person-based identifier exists, linkage can be achieved by simply merging datasets on the identifier. However, when a person-based identifier does not exist, then some other form of data matching or record linkage is required for integrating data.

Gill 2001

Hopf 2014

Haarbrandt 2016 [28]

Hu et al., 2007 [17]

Jones 2012

Elysee 2017 [29]

Akhlaq 2016 [15]

Dixon 2010 [33]

Esmaeilzadeh 2016 [34]

Esmaeilzadeh 2017 [35]

Fontaine 2010 [36]

Hopf 2014 [38]

Kash 2017 [39]

Mastebroek 2014 [27]

Parker 2016 [42]

Rahurkar 2015 [44]

Rudin 2014 [45]

Sadoughi 2018 [46]

Vest 2012 [48]

Where multiple studies used a similar definition, the review authors synthesised the data from similar definitions into the composite definition for each term, as presented in this table

- Data harmonisation occurs when there is an increasing availability of electronic data that can be pooled together using unique patient identifiers.

- Different types of data can be linked and shared such as individual patient clinical, pharmacy and laboratory data, health care utilisation and cost data, and personnel-related data.

- Electronic data required for DH processes can be found within and across different departments and institutions at facility, district, regional and national levels.
- A data harmonisation process consists of different types of technical activities such as identifying, reviewing, matching, defining, redefining, standardising, merging, linking, merging and formatting data.

- DH interventions or activities are defined according to a specific scope and purpose such as disease surveillance, monitoring of long-term outcomes, screening for adverse events, geographic area, secondary data use and data display mechanisms (data marts or dashboards). 


\section{Components and processes of data harmonisation}

To synthesise key components and processes of $\mathrm{DH}$ interventions (Objective 2(b)) we sampled 5 from the 61 studies identified for Objective 2 and 3. We selected 5 studies [16, 17, 29-31] based on the conceptual descriptions and visual illustrations of their $\mathrm{DH}$ interventions (See Table 4).

The conceptual description by [30], comes closest to a comprehensive conceptual model of a DH intervention, illustrating different types of data, different levels of the health care system (e.g. clinics and hospitals), the multiple processes of exchanging data, the multiple directions in exchange of data, and the key role of the unique patient identifier in enabling the DH process [30]. In the next model, Boyd et al. [16] and Santos et al. [31] both lay out the technical processes involved in the linkage process of different databases, but Santos et al. specifically focuses on linking data required for individual patient clinical management into a central repository. Lastly, Elysee et al. [29] and $\mathrm{Hu}$ et al. [17] describe DH interventions with different purposes, that is, medication reconciliation and disease outbreak surveillance respectively.

These conceptual models of $\mathrm{DH}$ interventions and activities highlight that there are various steps involved in the integration of databases and in the transformation of data into useable formats. Integrating databases means bringing together data of the same individual from within and between different electronic databases, through various activities involving identifying, reviewing, matching, redefining and standardising data $[1,16]$. Once data is harmonised, it can be categorised by various criteria of interest, such as geographic area or disease or patient population, and transformed into different formats such as graphs, tables or dashboards to make it easier for users to access and use the information [28]. There may be different ways that the data is harmonised; in some studies, DH is described as a linear and onedirectional process, while other studies described it as an iterative and multi-directional process.

\section{The relationship between data harmonisation and health management decision-making}

We sampled 9 studies from the 61 studies (identified for Objective 2 and 3 ) that provide an explanation of the relationship between DH and health management decision-making. These 9 studies were selected because they referred to the intended benefit, or directly referred to the relationship between DH and health management decision-making. We present extracts of explanations of the relationship in Table 5. According to Eylsee et al. [29] (the study providing the most detail), there is a positive relationship between increased availability of
Table 4 Concepts of data harmonisation interventions and processes

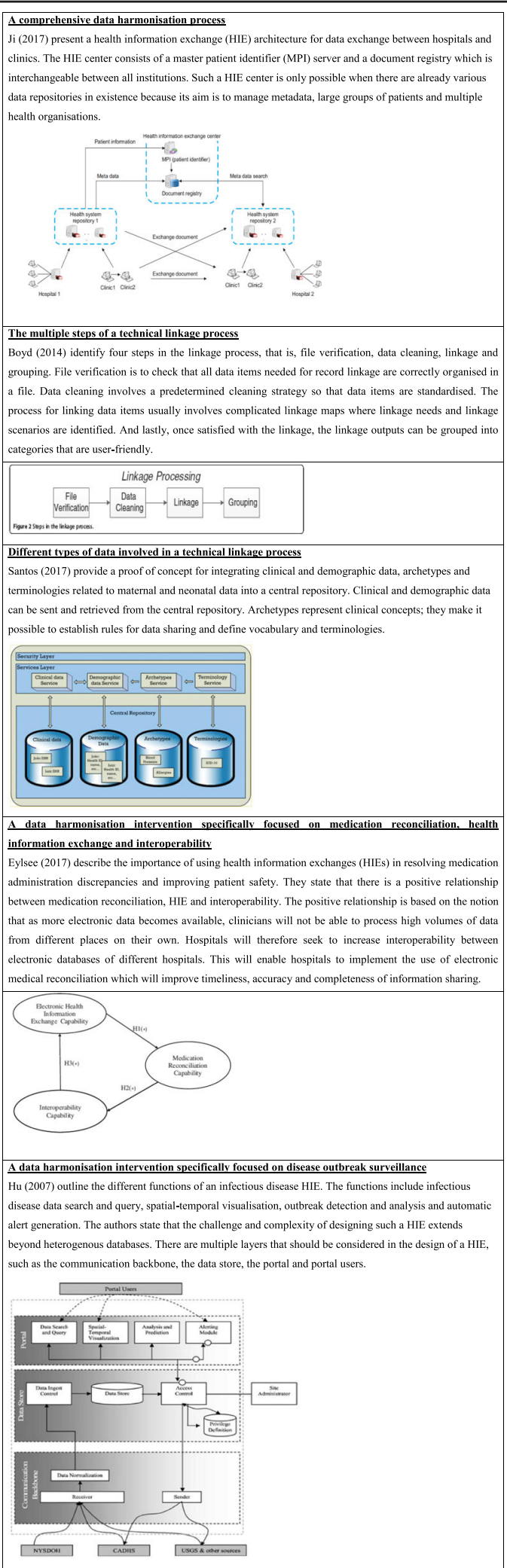

The table presents the different conceptual models of data harmonisation and the review authors provide a summary of how key components and processes were described by the authors of these models 
electronic data sets and the ability of clinicians to deal with high volumes of data. This necessitates interoperability between electronic databases at different hospitals, to improve timeliness, accuracy, and completeness of information sharing. According to Ji, Boyd, Santos and $\mathrm{Hu}$ the main benefit of $\mathrm{DH}$ is health management decision-making, including clinical decisionmaking [16, 17, 30, 31]. Across the studies, there is agreement that $\mathrm{DH}$ interventions make it possible for health providers to use data over time and across organisations to support clinical management decision-making. There is acknowledgement that DH interventions were sometimes unable to deal effectively with inconsistencies, incompleteness, and poor quality of data.

From the 9 studies, we identified three types of health management decision-making that $\mathrm{DH}$ contributed to. These are:

- Clinical decision-making for individual patient clinical management or clinical support and quality improvement tools

- Operational and strategic decision-making for health system managers and policy-makers

- Population-level decision-making for disease surveillance and outbreak management
The first level involves frontline clinicians being able to access their patients' medical information and treatment data and timelines (datasets of longitudinal, clinically relevant individual-level data) through $\mathrm{DH}$ interventions. In these situations, DH can make it easier for frontline clinicians to develop tools for reminding them about patients' performance in treatment and care services as well as help them improve the quality of health care services. At the operational and strategic decision-making level, DH interventions have the potential to support high-level health managers in decisionmaking involving a wide network of stakeholders (consumers, patients and professionals). Lastly, disease surveillance and outbreak management decision-making rely on harmonised data to plan, monitor and evaluate population-level interventions.

\section{Discussion}

\section{Synthesis of findings}

This scoping review aimed to provide an overview of the key characteristics of DH studies, identify definitions, alternative terms, components, and processes of DH interventions, and provide explanations of the relationship between $\mathrm{DH}$ and health management decision-making. Of the 181 studies that at a minimum provided a definition or description of a DH intervention or activity, 86 were primary quantitative studies, 151 were studies

Table 5 The relationship between DH interventions and health management decision-making

Cimino 2014 [21] "Data completeness: A promise of HIEs is to use consolidated information over time and across providers to
improve medical decision-making for the patient. When presenting a medical timeline for a patient, how
does a provider know whether the HIE presentation of history is missing information? The consequences to
patients can be devastating."
"... community-based approach to establish a common pathway based on common data standards to
facilitate the incorporation of interoperable, clinically useful genetic or genomic information and analytical
tools into EHRs to support clinical decision-making for the clinician and consumer."
"... the exchanges going beyond core clinical data exchange activities that give physicians access to data at
the point of care to offering physicians clinical decision support, reminders and other quality
improvement tools aimed at individual patients."

Kuperman 2013 [40] "Ideally, a physician would have access to complete, accurate and timely patient data to support optimal decision making. Health information exchange capabilities will reduce the extent of data fragmentation but will not eliminate it entirely."

Politi 2014 [41] "In this scenario, an HIE system is likely to have a significant impact on clinical decision making if information is readily accessible; the need for rapid decisions might render the scrutiny of an HIE system impractical."

Vest 2010 [43] $\quad$ "The anticipated benefits of more data to inform physician decision making, sparing patients of needless tests, helping organization identify inappropriately managed patients, and improving the health of the public will only be achieved by HIE that does not exclude providers in an area, limit what data elements are available, or restrict exchange to specific subpopulations."

Shapiro 2006 [47] "The goal of a nationwide health information network would be to deliver information to individuals- consumers, patients, and professionals - when and where they need it, so they can use this information to make informed decisions about health and health care."

Vest 2015 [49]

"Improved access to more comprehensive information may support decision-making, inform providers of additional medications or allegories, and help avoid repeated or duplicate testing."

Zaiden 2015 [50] "Combined with data mining and statistical analysis tools, these repositories of health information can greatly advance medical knowledge, healthcare quality, and good strategic management."

The review authors directly quoted text from the primary studies where a description of the link between data harmonisation and health management decisionmaking was provided 
conducted in the USA, and 128 were aimed at improving frontline level health services.

A key finding is that 'Health information exchange' or HIE, was the term most frequently used in the literature, especially for studies for the USA. Other terms used were data harmonisation, record linkage, data linkage, data warehousing, data sharing, and data interoperability. Terms like data harmonisation and data warehousing seem to describe a more comprehensive approach to $\mathrm{DH}$ interventions (involving both data production and data utilisation aspects), whereas terms like record and data linkage described specific activities within health information exchange. The term data interoperability focuses on the technical aspects that allows for different electronic databases to be linked and for data to be integrated, which allows for synthesis and analysis of health information. Even though different studies used different terms, there was consensus that DH is a useful tool for health management decision making and can support improvements in patient and health system outcomes.

We identified nine characteristics of DH interventions and activities. Using these nine characteristics, DH can be summarised as a process that aims to integrate two or more electronic databases, it involves different types of data captured within and across various institutions at different health care system levels, and varying activities are required to pool together data using unique patient identifiers for the purpose of providing information support for health management decision-making. The review identified three types of health management decisionmaking that DH contributed to: (a) clinical decisionmaking for individual patient management, clinical support and quality improvement tools; (b) operational and strategic decision-making for health system managers and policy-makers; and (c) population-level decision-making for disease surveillance and outbreak management.

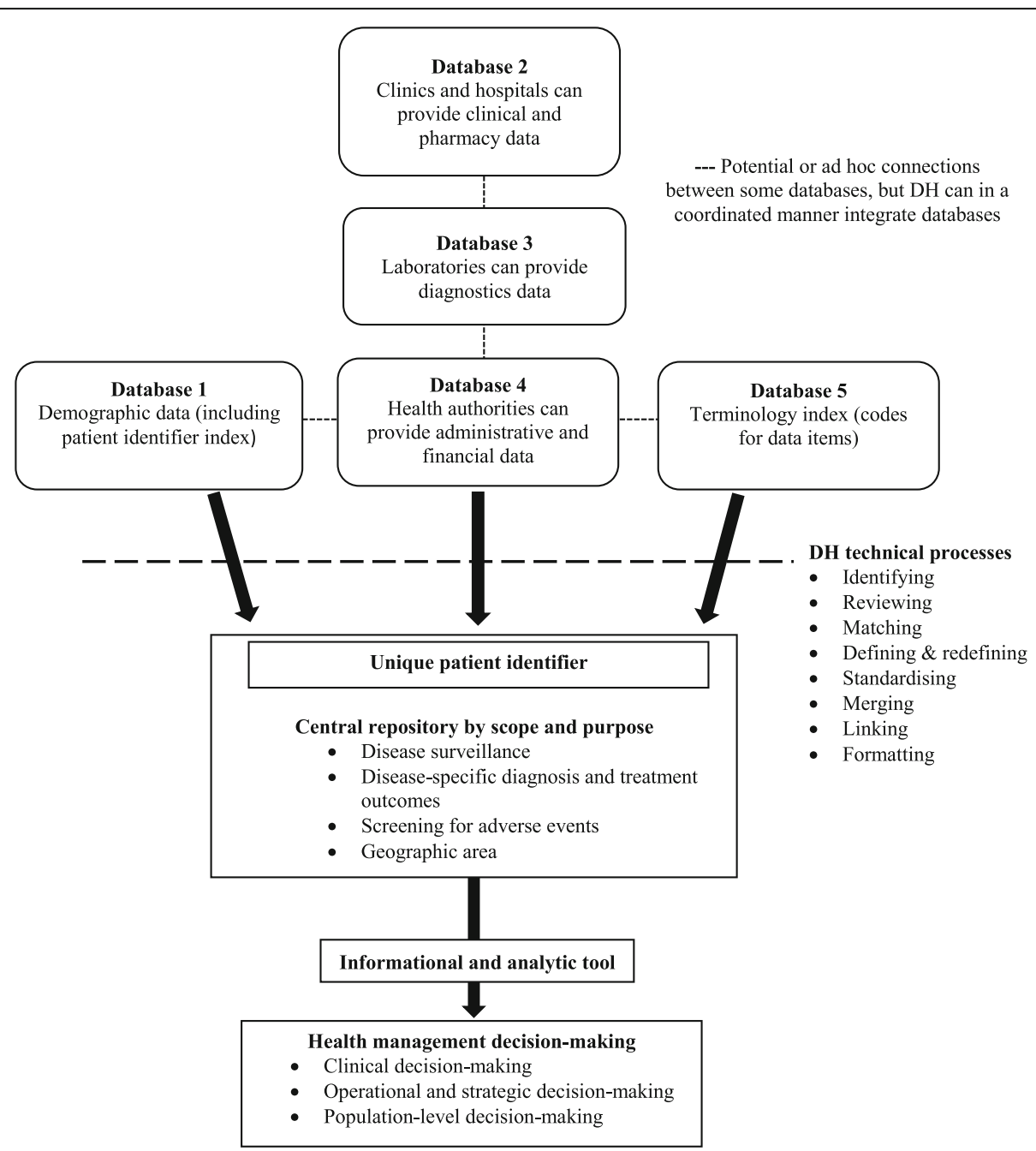

Fig. 3 A concept map of data harmonisation and its relationship to health management decision-making 
Drawing on the definitions and the conceptual models of DH identified in this review, we developed a concept map (see Fig. 3) to explain how different aspects of DH interventions and activities work together to support health management decision-making. The concept map consists of different types of databases ( 1 to 5 ) containing different types of data such as demographic, clinical, pharmacy, laboratory, administrative and financial, and terminology data. A technical process involving different types of activities (such as matching, merging and linking) takes place to integrate the different types of data using a unique patient identifier. The central repository, where the data is harmonised, is defined according to specific criteria such as a geographic area or disease outcomes. The data kept in the repository should be accessible to data users, who can then use this harmonised data as an information and analytic tool to support health management decisionmaking for clinical, operational, strategic, and or population-level decision-making.

\section{Study limitations}

There are two main differences between the published protocol and this scoping review. We did not search the Global Health database as planned; we realised late that none of the reviewers had permissions to access the database and gaining access was not affordable. We did however manage to search at least three electronic databases, as is the convention in reviews [23]. Due to the large volume of studies included for full-text screening, it was not feasible to conduct the full text screening in duplicate as planned. The first reviewer (BS) assessed all full-texts and then the second reviewer $(\mathrm{AH})$ verified the decisions of the first reviewer in a third of the included studies, which allowed for additional quality checks.

There are two main limitations of the review. Firstly, we restricted our literature search to English. We did not have the resources required for reviewing nonEnglish studies. Most studies identified were from the USA, but it is possible that studies from other nonEnglish speaking, high-income countries with extensive electronic health systems (such as France) may have been missed. Secondly, although sampling aimed to identify variety, comprehensiveness and meaningfulness of the definitions and explanations, there is a possibility that due to sampling, we may have missed relevant studies for Objectives 2 and 3.

\section{Implications for research and practice}

There is a need to understand what DH interventions and activities are comprised of in diverse settings and contexts, especially in LMICs. There were fewer studies from LMICs, which may be due to a lower prevalence of electronic health information systems in those settings. Nevertheless, DH interventions hold promise for improving the informational support in LMICs; studies in these contexts could usefully expand the evidence base.

The review highlights the importance of providing detailed descriptions of DH interventions, to allow for better comparisons and to improve the transferability of study results. Additionally, many resources are spent on the technical development of DH projects, with the implicit assumption that this will provide the informational and analytic support for health management decisionmaking, but this assumption is seldom tested in the research. There is a need for qualitative research on the health system factors of implementing $\mathrm{DH}$ and for formative work to inform design of DH interventions. Finally, primary research and evidence synthesis of the experiences of key stakeholders involved (implementers and users of harmonised data) would improve our understanding of the causal mechanisms between data harmonisation and health systems strengthening.

\section{Conclusion}

The review aimed to widen our understanding about the range of definitions, components and processes of $\mathrm{DH}$ interventions, and how it can contribute to health management decision-making. Most studies of DH interventions and activities were conducted in high-income settings and used the term 'health information exchange'. The review described the processes, technical activities, types of data, mechanisms for integrating data, and purpose of the $\mathrm{DH}$ interventions. $\mathrm{DH}$ interventions contributed to three types of health management decision-making, that is, clinical decision-making, operational and strategic decision-making, and populationlevel surveillance decision-making. We provided a concept map of the components of DH and make recommendations for future research.

\section{Abbreviations \\ $\mathrm{DH}$ : Data harmonisation; RHISs: Routine health information systems; LMICs: Low- and middle-income countries; MeSH: Medical Subject Headings; HIS: Health information system; IT: Information technology; HIE: Health information exchange}

\section{Acknowledgements \\ We would like to thank Ms. Gill Morgan, University of Cape Town, who assisted with developing the search strategy.}

\section{Authors' contributions}

BS was involved in all the tasks of conducting the scoping review. She drafted the manuscript with help from CC and NL. AH contributed to searching, screening and data extraction processes. All authors reviewed and approved the final manuscript before final submission for peer review.

\section{Funding}

Time to write this paper was supported by the US National Institute of Mental Health [grant number 1R01 MH106600] and the South African Medical Research Council (SAMRC). The content of this paper is solely the responsibility of the authors and does not necessarily represent the official views of the US National Institutes of Health or the SAMRC. 


\section{Availability of data and materials}

The datasets used and/or analysed during the current study are available from the corresponding author on reasonable request.

\section{Ethics approval and consent to participate}

Not applicable.

\section{Consent for publication}

Not applicable.

\section{Competing interests}

The authors declare that they have no competing interests.

\section{Author details}

${ }^{1}$ Cochrane South Africa, South African Medical Research Council, Francie Van Zijl Dr, Parow Valley, Cape Town 7501, South Africa. ${ }^{2}$ Division of Social and Behavioural Sciences, School of Public Health and Family Medicine, University of Cape Town, Cape Town, South Africa. ${ }^{3}$ Department of Public Health Sciences, University of Virginia, Charlottesville, USA. ${ }^{4}$ Department of Epidemiology, School of Public Health, Brown University, Providence, USA. ${ }^{5}$ Health Systems Research Unit, South African Medical Research Council, Cape Town, South Africa.

Received: 23 April 2020 Accepted: 12 August 2020

Published online: 14 September 2020

\section{References}

1. Liu D, et al. Harmonization of health data at national level: a pilot study in China. Int J Med Inform. 2010;79(6):450 https://doi.org/10.1016/j.jimedinf. 2010.03.002.

2. Nutley T, Reynolds $H$. Improving the use of health data for health system strengthening. Glob Health Action. 2013;6(1):20001 https://doi.org/10.3402/ gha.v6i0.20001.

3. Lippeveld T. Routine health information systems: the glue of a unified health system. In: Keynote address at the Workshop on Issues and Innovation in Routine Health Information in Developing Countries. Potomac; 2001.

4. World Health Organization. Everybody's business-strengthening health systems to improve health outcomes: WHO's framework for action; 2007. Retrieved from https://www.who.int/healthsystems/strategy/everybodys_ business.pdf?ua=1.

5. World Health Organization. Country health information systems assessments: overview and lessons learnt. 2012.

6. Heywood A, Boone D. Guidelines for data management standards in routine health information systems. Measure Evaluation. 2015; Retrieved from https://www.measureevaluation.org/resources/publications/ms-15-99.

7. Karuri J, et al. DHIS2: the tool to improve health data demand and use in Kenya. J Health Informatics Dev Countries. 2014;8(1):38 Retrieved from https://jhidc.org/index.php/jhidc/article/view/113.

8. Harrison Ml, et al. Unintended consequences of information technologies in health care-an interactive sociotechnical analysis. J Am Med Inform Assoc. 2007;14(5):542 https://doi.org/10.1197/jamia.M2384.

9. Olmen JV, et al. The Health System Dynamics Framework: The introduction of an analytical model for health system analysis and its application to two case-studies. Health Culture Soc. 2012;2(1):1 Retrieved from https://hcs.pitt. edu/ojs/index.php/hcs/article/view/71/0.

10. Sittig DF, Singh $\mathrm{H}$. A new sociotechnical model for studying health information technology in complex adaptive healthcare systems. BMJ Qual Saf. 2010;19(Suppl 3):i68 https://doi.org/10.1136/qshc.2010.042085.

11. Plsek, P. Complexity and the adoption of innovation in health care. Accelerating Quality Improvement in Health Care: Strategies to Accelerate the Diffusion of Evidence-Based Innovations. National Institute for Healthcare Management Foundation and National Committee for Quality in Health Care. 2003. Retrieved from https://www.nihcm.org/pdf/Plsek.pdf.

12. Cresswell KM, Sheikh A. Undertaking sociotechnical evaluations of health information technologies. J Innovation Health Informatics. 2014;21(2):78 https://doi.org/10.14236/jhi.v21i2.54

13. Cresswell KM, et al. Ten key considerations for the successful optimization of large-scale health information technology. J Am Med Inform Assoc. 2016; 24(1):182 https://doi.org/10.1093/jamia/ocw037.
14. Fichtinger $A$, et al. Data harmonisation put into practice by the HUMBOLDT project. Int J Spatial Data Infrastructures Res. 2011;6:234 https://doi.org/10. 2902/ijsdir.v6i0.191.

15. Akhlaq A, et al. Barriers and facilitators to health information exchange in low- and middle-income country settings: a systematic review. Health Policy Plan. 2016;31(9):1310 https://doi.org/10.1093/heapol/czw056.

16. Boyd $\mathrm{JH}$, et al. Technical challenges of providing record linkage services for research. BMC Med Informatics Decision Making. 2014;14(1):23 https://doi. org/10.1186/1472-6947-14-23.

17. Hu PJ, et al. System for infectious disease information sharing and analysis: design and evaluation. IEEE Trans Inf Technol Biomed. 2007a;11(4):483 https://doi.org/10.1109/titb.2007.893286.

18. Aqil A, et al. PRISM framework: a paradigm shift for designing, strengthening and evaluating routine health information systems. Health Policy Plan. 2009;24(3):217 https://doi.org/10.1093/heapol/czp010.

19. Arksey H, O'Malley L. Scoping studies: towards a methodological framework. Int J Soc Res Methodol. 2005;8(1):19 https://doi.org/10.1080/ 1364557032000119616.

20. Schmidt B-M, et al. Defining and conceptualising data harmonisation: a scoping review protocol. Syst Rev. 2018;7(1):226 https://doi.org/10.1186/ s13643-018-0890-7.

21. Cimino JJ, et al. Consumer-mediated health information exchanges: the 2012 ACMI debate. J Biomed Inform. 2014;48(2014):5 https://doi.org/10. 1016/j.jbi.2014.02.009.

22. Tricco AC, et al. A scoping review on the conduct and reporting of scoping reviews. BMC Med Res Methodol. 2016;16(1):15 https://doi.org/10.1016/j.jbi. 2014.02.009.

23. Cochrane Effective Practice and Organisation of Care (EPOC) Group. EPOC Qualitative Evidence Syntheses: protocol template. 2018. Retrieved from https://epoc.cochrane.org/news/qualitative-evidence-synthesis-template.

24. Suri H. Purposeful sampling in qualitative research synthesis. Qual Res J. 2011;11(2):63 https://doi.org/10.3316/QRJ1102063.

25. Popay J, et al. Guidance on the conduct of narrative synthesis in systematic reviews. In: A product from the ESRC methods programme; 2006. https:// doi.org/10.13140/2.1.1018.4643.

26. Leon $\mathrm{N}$, et al. Routine Health Information System (RHIS) interventions to improve health systems management. Cochrane Database Syst Rev. 2015; 12(CD012012):1 https://doi.org/10.1002/14651858.CD012012.

27. Mastebroek M, Naaldenberg J, Lagro-Janssen AL, van Schrojenstein Lantman de Valk H. Health information exchange in general practice care for people with intellectual disabilities: a qualitative review of the literature. Res Dev Disabil. 2014;35(9):1978-87 https://doi.org/10.1016/j.ridd.2014.04.029.

28. Haarbrandt BE, et al. Automated population of an i2b2 clinical data warehouse from an openEHR-based data repository. J Biomed Inform. 2016; 63(2016):277 https://doi.org/10.1016/j.jbi.2016.08.007.

29. Elysee $\mathrm{GJ}$, et al. An observational study of the relationship between meaningful use-based electronic health information exchange, interoperability, and medication reconciliation capabilities. Medicine (Baltimore). 2017;96(41):e8274 https://doi.org/10.1097/MD. 0000000000008274.

30. Ji $\mathrm{H}$, et al. Technology and policy challenges in the adoption and operation of health information exchange systems. Adv Health Care Manag. 2017; 23(4):314 https://doi.org/10.4258/hir.2017.23.4.314.

31. Santos MR, et al. Health information exchange for continuity of maternal and neonatal care supporting: a proof-of-concept based on ISO standard. Applied Clin Informatics. 2017;8(4):1082 https://doi.org/10. 4338/ACl-2017-06-RA-0106.

32. Downs SM, van Dyck PC, Rinaldo P, McDonald C, Howell RR, Zuckerman A, Downing $G$. Improving newborn screening laboratory test ordering and result reporting using health information exchange. J Am Med Inform Assoc. 2010;17(1):13-8 https://doi.org/10.1197/jamia.M3295.

33. Dixon BE, Zafar A, Overhage JM. A Framework for evaluating the costs, effort, and value of nationwide health information exchange. J Am Med Inform Assoc. 2010;17(3):295-301 https://doi.org/10.1136/jamia.2009.000570.

34. Esmaeilzadeh $\mathrm{P}$, Sambasivan M. Health Information Exchange (HIE): A literature review, assimilation pattern and a proposed classification for a new policy approach. J Biomed Inform. 2016;64:74-86. https://doi.org/10. 1016/j.jbi.2016.09.011.

35. Esmaeilzadeh P, Sambasivan M. Patients' support for health information exchange: a literature review and classification of key factors. BMC Med Inform Decis Mak. 2017;17:33 https://doi.org/10.1186/s12911-017-0436-2. 
36. Fontaine P, Ross SE, Zink T, Schilling LM. Systematic review of health information exchange in primary care practices. J Am Board Fam Med. 2010;23(5):655-70. https://doi.org/10.3122/jabfm.2010.05.090192.

37. Grossman JM, Kushner KL, November EA. Creating sustainable local health information exchanges: can barriers to stakeholder participation be overcome? Res Brief. 2008;2:1-12.

38. Hopf YM, Bond C, Francis J, Haughney J, Helms PJ. Views of healthcare professionals to linkage of routinely collected healthcare data: a systematic literature review. J Am Med Inform Assoc. https://doi.org/10.1136/amiajnl2012-001575.

39. Kash BA, Baek J, Davis E, Champagne-Langabeer T, Langabeer JR 2nd. Review of successful hospital readmission reduction strategies and the role of health information exchange. Int J Med Inform. 2017;104:97-104 https:// doi.org/10.1016/j.jimedinf.2017.05.012.

40. Kuperman GJ, McGowan JJ. Potential unintended consequences of health information exchange. J Gen Intern Med. 2013;28(12):1663-6 https://doi.org/ 10.1007/s11606-012-2313-0.

41. Politi L, Codish S, Sagy I, Fink L. Use patterns of health information exchange through a multidimensional lens: conceptual framework and empirical validation. J Biomed Inform. 2014;52:212-21 https://doi.org/10. 1016/j.jbi.2014.07.003.

42. Parker C, Weiner M, Reeves M. Health information exchanges--Unfulfilled promise as a data source for clinical research. Int J Med Inform. 2016;87:1-9 https://doi.org/10.1016/j.jjmedinf.2015.12.005.

43. Vest JR, Gamm LD. Health information exchange: persistent challenges and new strategies. J Am Med Inform Assoc. 2010;17(3):288-94 https://doi.org/ 10.1136/jamia.2010.003673.

44. Rahurkar S, Vest JR, Menachemi N. Despite the spread of health information exchange, there is little evidence of its impact on cost,use, and quality of care. Health Aff. 2015;34(3):477-83 https://doi.org/10.1377/hlthaff.2014.0729.

45. Rudin RS, Motala A, Goldzweig CL, Shekelle PG. Usage and effect of health information exchange: a systematic review. Ann Intern Med. 2014;161(11): 803-11 https://doi.org/10.7326/M14-0877.

46. Sadoughi F, Nasiri S, Ahmadi H. The impact of health information exchange on healthcare quality and cost-effectiveness: A systematic literature review. Comput Methods Prog Biomed. 2018;161:209-32 https://doi.org/10.1016/j. cmpb.2018.04.023.

47. Shapiro JS, Kannry J, Lipton M, Goldberg E, Conocenti P, Stuard S, Wyatt BM, Kuperman G. Approaches to Patient Health Information Exchange and Their Impact on Emergency Medicine. Ann Emerg Health. 2006; https://doi.org/ 10.1016/j.annemergmed.2006.03.032.

48. Vest JR, Jasperson S. How are health professionals using health information exchange systems? Measuring usage for evaluation and system improvement. J Med Syst. 2012;36(5):3195-204 https://doi.org/10.1007/ s10916-011-9810-2.

49. Vest JR, Abramson E. Organizational Uses of Health Information Exchange to Change Cost and Utilization Outcomes: A Typology from a Multi-Site Qualitative Analysis. AMIA Annu Symp Proc. 2015:1260-8.

50. Zaidan BB, Haiqi A, Zaidan AA, Abdulnabi M, Mat Kiah ML, Muzamel H. A Security Framework for Nationwide Health Information Exchange based on Telehealth Strategy. J Med Syst. 2015;39(5):235. https://doi.org/10.1007/ s10916-015-0235-1.

\section{Publisher's Note}

Springer Nature remains neutral with regard to jurisdictional claims in published maps and institutional affiliations.

Ready to submit your research? Choose BMC and benefit from:

- fast, convenient online submission

- thorough peer review by experienced researchers in your field

- rapid publication on acceptance

- support for research data, including large and complex data types

- gold Open Access which fosters wider collaboration and increased citations

- maximum visibility for your research: over $100 \mathrm{M}$ website views per year

At BMC, research is always in progress.

Learn more biomedcentral.com/submissions 